\title{
Risk factors for in-patient admission among adults with intellectual disability and autism: investigation of electronic clinical records
}

Rory Sheehan, Jennifer Mutch, Louise Marston, David Osborn and Angela Hassiotis

Adults with intellectual disability or autism are at risk of psychiatric admission which carries personal, social and economic costs. We identified 654 adults with intellectual disability or autism in the electronic clinical records of one mental health trust. We investigated the demographic and clinical factors associated with admission and readmission after discharge. Young male patients with intellectual disability, schizophrenia and previous admissions are most at risk of the former, whereas affective and personality disorders predict the latter. Both community intellectual disability services and mental health crisis care must focus on providing effective support for those patients.

\section{Keywords}

Admissions; autism; intellectual disability; crisis care; risk.

\section{Copyright and usage}

(C) The Author(s), 2020. Published by Cambridge University Press on behalf of the Royal College of Psychiatrists. This is an Open Access article, distributed under the terms of the Creative Commons Attribution licence (http://creativecommons.org/ licenses/by/4.0/), which permits unrestricted re-use, distribution, and reproduction in any medium, provided the original work is properly cited.
Both intellectual disability and autism are associated with significant additional psychiatric morbidity. ${ }^{1,2}$ Poor understanding of mental disorder in these groups, the presence of challenging behaviour, and lack of access to appropriate community-based care and support may lead to unnecessary or prolonged admissions to psychiatric in-patient care. Reducing inappropriate psychiatric admissions is a cornerstone of current UK healthcare policy, and a priority for people with intellectual disability, autism and their social networks. ${ }^{3}$

NHS England mandate that clinical commissioning groups maintain 'at risk of admission' registers that identify those at greatest risk of hospital admission and ensure these individuals are prioritised and supported effectively at times of crisis. ${ }^{4}$ Indicators for inclusion on the register are not well established and vary locally.

We used patient-level data to explore associations with inpatient admission, and with readmission following discharge, of adults with intellectual disability and/or autism accessing care from a mental health trust.

\section{Method}

\section{Data source}

Data were obtained from the Camden and Islington NHS Foundation Trust electronic clinical record, using the Clinical Record Interactive Search (CRIS) tool. The Trust provides services to an ethnically and socioeconomically diverse population of approximately 500000 people. Their electronic clinical record contains anonymised information of over 130000 adults, and the Trust only admits mentally ill individuals who reside within its area boundaries and only those with mild-to-moderate intellectual disability. ${ }^{5,6}$ Data can be extracted from both structured fields and from the free-text records, applying natural language processing (NLP) algorithms. The authors assert that all procedures contributing to this work comply with the ethical standards of the relevant national and institutional committees on human experimentation and with the Helsinki Declaration of 1975, as revised in 2008. All procedures involving human subjects/patients were approved by the East of England-Cambridge Central Research Ethics
Committee (approval number 14/EE/0177). The specific project was approved by the Camden and Islington CRIS oversight panel.

\section{Study cohort}

Adults ( $\geq 18$ years) with ICD-10 diagnostic codes F70-F79 (mental retardation) and/or F84 (pervasive developmental disorders) who had accessed secondary mental healthcare within the cohort period were included. In addition, NLP was used to explore the clinical records (assessment and progress notes) for terms related to intellectual disability and/or autism. Eligibility for inclusion of those identified via NLP was validated by one of the authors (J.M.), with queries resolved by discussion with another author (A.H.). Entry to the cohort was 1 January 2009, the date of turning 18 years or discharge from hospital (if in hospital on 1 January 2009), whichever was latest. Exit from the cohort was 30 November 2018, date of death or admission to hospital (if in hospital on 30 November 2018), whichever was earliest.

\section{Outcomes and covariates}

The main outcome was first admission to psychiatric in-patient care during the cohort period. The secondary outcome was readmission to the acute care pathway (psychiatric in-patient care or crisis team intervention) within 12 months following discharge from the first admission in the cohort period.

We extracted data on diagnostic group (intellectual disability with or without autism, and autism only); age at cohort entry; gender; ethnicity; diagnosis of mental illness (coded according to the ICD $-10^{7}$ ); social deprivation (estimated with the Index of Multiple Deprivation ${ }^{8}$ ); and presence of internalising or externalising challenging behaviour (measured with the Health of The Nation Outcome Scales $^{9}$ ) for each individual.

\section{Statistical analysis}

All categorical variables were analysed with chi-squared tests, apart from eating disorders, which was analysed with Fisher's exact test because of the small number in the cells that violated the test assumptions. Continuous variables were analysed with $t$-tests, 
with the exception of median duration of admission, which was analysed with the Mann-Whitney $U$-test because of skewness. Logistic regression was used to explore associations between in-patient admission and demographic and clinical variables and discharge to a destination other than a person's usual residence. We plotted time to readmission to the acute care pathway within 12 months of discharge, using a Kaplan-Meier plot, and utilised Cox regression to determine differences in time to readmission between the groups controlling for confounders. We carried out multiple imputation using chained equations for missing data for the analyses related to first admission. We calculated the rate of in-patient admissions during each year of the cohort and tested the change in the rate over time by using Poisson regression, with person-years as the exposure. Data were analysed with Stata version 16 for Windows.

\section{Results}

\section{Profile of people using services}

We identified 339 adults with intellectual disability with or without autism and 315 adults with autism only who accessed mental healthcare services between January 2009 and November 2018. Each person contributed an average of 7.94 years follow-up.

The autism only group were younger and included a greater proportion of males than the intellectual disability group. Adults with intellectual disability were more likely to have been diagnosed with schizophrenia spectrum disorders $(47.8 \%$ v. $18.1 \%, P<0.001)$, whereas a greater proportion in the autism-only group had a diagnosis of anxiety disorder $(11.2 \%$ v. $24.9 \%, P<0.001)$ (Table 1$)$. Approximately a third of the total sample were admitted to hospital at least once during the cohort period.

\section{Admission and discharge within the cohort period}

The median duration of first admission in the cohort period was 34 days (interquartile range, 11-95 days) in the intellectual disability group, and 27 days (interquartile range, 14-58 days) in the autism-only group $(P=0.211)$. Likelihood of psychiatric admission was higher in those with intellectual disability (adjusted odds ratio (aOR), 4.00; 95\% CI 2.41-6.63), males (aOR, 2.28; 95\% CI 1.39-3.75), younger age (aOR, $0.98 ; 95 \% \mathrm{CI} 0.97-1.00)$ and in those with a diagnosis of schizophrenia spectrum disorder (aOR, 5.08; 95\% CI 3.00-8.61), affective disorder (aOR, 2.23; 95\% CI 1.29-3.83), personality disorder (aOR, 1.94; 95\% CI 1.02-3.68) and record of a previous admission (aOR, 2.18; 95\% CI 1.17-4.05).

Discharge destination after first admission was usual residence for $57.4 \%$ of adults with intellectual disability and $78.7 \%$ for the autism-only group. Discharge to a place other than usual residence was associated with longer duration of admission (aOR, 1.01; 95\% CI 1.00-1.01), and having externalising or internalising challenging behaviours (aOR, 3.34; 95\% CI 0.65-17.13 and aOR, 9.87; 95\% CI $1.75-55.70$, respectively). Living in a more deprived area was associated with discharge to usual residence (aOR, 0.90 ; 95\% CI 0.83-0.97).

There was no statistically significant change in the rate of admissions over time, including the years 2014-2018, during which the new policy initiative 'Transforming Care' was implemented in England. ${ }^{10}$

\section{Re-entry to the acute care pathway}

A total of $40 \%$ of those who had an admission during the cohort period were readmitted or had contact with mental health crisis services within 12 months of discharge, mostly within the first 6 months after discharge (see Supplementary Fig. 1 available at https://doi.org/10.1192/bjo.2020.135). Those with autism only had a greater risk of readmission, although this difference was not statistically significant (aOR, 1.43; 95\% CI 0.66-3.05). Comorbid diagnoses of affective disorder or personality disorder were significantly associated with readmission (aOR, 3.11; 95\% CI 1.34-7.23 and aOR, 8.28; 95\% CI 2.85-24.04, respectively).

\section{Discussion}

In summary, we found that young male patients with intellectual disability, schizophrenia spectrum disorder and previous admissions are most at risk of a new admission to psychiatric in-patient care, whereas only affective and personality disorders predict readmission. Almost half of those with an intellectual disability are unable to return to their original accommodation following discharge. Both adults with intellectual disabilities or autism only are vulnerable to admission, but each group may have differential needs and both are likely to be in contact with acute mental health services soon after discharge. The reasons behind the pattern of readmissions following contacts with crisis teams merits further exploration. It may be that patient characteristics may affect outcome, or that potentially patients are discharged with a degree of unmet need or that their needs are not fully understood at the point of contact with crisis professionals. As community intellectual disability services are required to maintain a dynamic register of patients 'at risk of admission', our findings can inform the effective use of the register by guiding the provision of services directed to those patients. These must include proactive liaison with crisis care or home treatment teams, and availability of staff with experience in working with those individuals at the time of assessment.

Being discharged to a place other than their usual residence demonstrates the potential for in-patient admission to disrupt a person's life course. Both internalising and externalising behaviours were strongly associated with the original residence being deemed unsuitable to support the person following discharge in about half of adults with intellectual disability. This finding underlines the need for responsive specialist behaviour community services for patients with complex presentations.

The cohort period includes the years spanning the innovations introduced as part of Transforming Care, including the introduction of Care and Treatment Reviews. ${ }^{10}$ It would appear that the initiation of such reviews has not influenced the rate of admissions of people with intellectual disability or with autism to psychiatric inpatient wards, at least in this area. This is in accord with NHS Digital data, which show that Transforming Care has not had the desired effect on reduction of admissions. ${ }^{11}$

This is the first study using routinely collected clinical adult mental health data to investigate the pattern of admissions of people with intellectual disability and with autism. The results are highly relevant in the context of changes in the care of this population group, which began with the launch of the White Paper 'Valuing People'12 in 2001. Valuing People promoted the use of general psychiatric services by people with intellectual disability and with autism who have mental ill health, but its implementation has not been fully appraised, and this group continue to experience health inequalities related to poor access to healthcare, which may extend to inappropriate in-patient admission.

The study also has limitations, including lack of inclusion of those people who were transferred to out-of-area facilities, for example, specialist hospitals. Psychiatric comorbidities could be attributed to reverse causality as those who are admitted are more likely to receive a mental health diagnosis because of increased contact. ${ }^{13}$ 


\begin{tabular}{|c|c|c|c|c|}
\hline Variable & Intellectual disability with/without autism $(n=339)$ & Autism only $(n=315)$ & Total sample $(n=654)$ & $P$-value \\
\hline \multicolumn{5}{|l|}{ Degree of intellectual disability } \\
\hline Mild & 114 & - & 114 & \\
\hline Moderate-to-severe & 16 & - & 16 & \\
\hline Missing/not recorded & 209 & - & 209 & \\
\hline Not applicable & - & 315 & 315 & \\
\hline \multicolumn{5}{|l|}{ Gender } \\
\hline Male & $177(52.2 \%)$ & $235(74.6 \%)$ & 412 (63.0\%) & $<0.001$ \\
\hline Female & $162(47.8 \%)$ & 80 (25.4\%) & $242(37.0 \%)$ & \\
\hline Age at cohort entry, mean (s.d.) & $35.8(14.9)$ & $30.5(13.27)$ & $33.2(14.4)$ & $<0.001$ \\
\hline \multicolumn{5}{|l|}{ Ethnic group } \\
\hline White & $205(60.5 \%)$ & $171(54.3 \%)$ & $376(57.5 \%)$ & 0.226 \\
\hline Not White & 112 (33.0\%) & 75 (23.8\%) & $187(28.6 \%)$ & \\
\hline Missing & $22(6.5 \%)$ & $69(21.9 \%)$ & $91(13.9 \%)$ & \\
\hline Index multiple deprivation, mean (s.d.) & $33.2(12.37)$ & $31.2(11.49)$ & $32.3(12.00)$ & \\
\hline Missing & 19 & 24 & 43 & 0.016 \\
\hline \multicolumn{5}{|l|}{ Died in cohort period } \\
\hline Yes & $20(5.9 \%)$ & $6(1.9 \%)$ & $26(4.0 \%)$ & 0.009 \\
\hline No & 319 (94.1\%) & 309 (98.1\%) & $628(96.0 \%)$ & \\
\hline \multicolumn{5}{|l|}{ Admitted in cohort period } \\
\hline Yes & 155 (45.7\%) & $61(19.4 \%)$ & 216 (33.0\%) & $<0.001$ \\
\hline No & $184(54.3 \%)$ & $254(80.6 \%)$ & 438 (67.0\%) & \\
\hline \multicolumn{5}{|l|}{ Crisis team contact in cohort period } \\
\hline Yes & $151(44.5 \%)$ & $108(34.3 \%)$ & $259(39.6 \%)$ & $<0.001$ \\
\hline No & $188(55.5 \%)$ & 207 (65.7\%) & $395(60.4 \%)$ & \\
\hline \multicolumn{5}{|l|}{ HONOS externalising } \\
\hline No problem & $132(38.9 \%)$ & $167(53.0 \%)$ & $299(45.7 \%)$ & $<0.001$ \\
\hline Problem & 109 (32.2\%) & $65(20.6 \%)$ & $174(26.6 \%)$ & \\
\hline Missing & 98 (28.9\%) & $83(26.3 \%)$ & $181(27.7 \%)$ & \\
\hline \multicolumn{5}{|l|}{ HoNOS internalising } \\
\hline No problem & 204 (60.2\%) & $186(59.0 \%)$ & $390(59.6 \%)$ & 0.201 \\
\hline Problem & 37 (10.9\%) & $46(14.6 \%)$ & $83(12.7 \%)$ & \\
\hline Missing & 98 (28.9\%) & $83(26.3 \%)$ & $181(27.7 \%)$ & \\
\hline \multicolumn{5}{|l|}{ Diagnosis } \\
\hline F00-F09 (dementia) & $22(6.5 \%)$ & $8(2.5 \%)$ & $30(4.6 \%)$ & 0.014 \\
\hline F10-F19 (drug/alcohol) & $37(14.7 \%)$ & $15(5.4 \%)$ & $52(9.9 \%)$ & $<0.001$ \\
\hline F20-F29 (SSD) & $120(47.8 \%)$ & 50 (18.1\%) & $170(32.2 \%)$ & $<0.001$ \\
\hline F30-F39 (mood) & 57 (22.7\%) & 57 (20.6\%) & $114(21.6 \%)$ & 0.552 \\
\hline F40-F48 (anxiety) & $28(11.2 \%)$ & $69(24.9 \%)$ & 97 (18.4\%) & $<0.001$ \\
\hline F50-F59 (eating disorders) & $2(0.8 \%)$ & $4(1.4 \%)$ & $6(1.1 \%)$ & 0.690 \\
\hline F60-F60 (personality disorder) & 39 (15.5\%) & $37(13.4 \%)$ & 76 (14.4\%) & 0.498 \\
\hline F90-F98 (childhood onset) & $11(4.4 \%)$ & $29(10.5 \%)$ & $40(7.6 \%)$ & 0.008 \\
\hline Missing any diagnosis & $138(40.7 \%)$ & $120(38.1 \%)$ & $258(39.4 \%)$ & \\
\hline \multicolumn{5}{|l|}{ Antipsychotic (ever prescribed) } \\
\hline Yes & $230(67.9 \%)$ & $112(35.6 \%)$ & $312(47.7 \%)$ & $<0.001$ \\
\hline No & 109 (32.2\%) & $203(64.4 \%)$ & $342(52.3 \%)$ & \\
\hline \multicolumn{5}{|l|}{ Other psychotropic (ever prescribed) } \\
\hline Yes & 254 (74.9\%) & $181(57.5 \%)$ & $435(66.5 \%)$ & $<0.001$ \\
\hline No & 85 (25.1\%) & $134(42.5 \%)$ & 219 (33.5\%) & \\
\hline \multicolumn{5}{|l|}{ Admitted to hospital before cohort entry } \\
\hline Yes & 65 (19.2\%) & $25(7.9 \%)$ & $90(13.8 \%)$ & $<0.001$ \\
\hline No & $274(80.8 \%)$ & 290 (92.1\%) & $564(86.2 \%)$ & \\
\hline Cohort contribution, years, mean (s.d.) & $8.28(1.53)$ & $7.57(2.30)$ & $7.94(1.97)$ & $<0.001$ \\
\hline
\end{tabular}

In conclusion, the development and maintenance of 'at risk of admission' registers with monthly reporting by clinical commissioning groups to NHS England ensures close scrutiny of outcomes for people with intellectual disability and/or autism who are acutely mentally ill. In order for the registers to be most effectively used, they should be accompanied by robust and clearly defined responses by community intellectual disability and crisis care teams, tailored to the most vulnerable patients. Despite early UK studies that failed to show efficacy, ${ }^{14}$ research outside the UK suggests that assertive outreach models may be adapted successfully for this population. ${ }^{15}$ Given how much is at stake in terms of poor quality of life and years spent in inpatient units, revisiting prevention of admissions and emergency care response is urgently needed.
Rory Sheehan (D), PhD MRCPsych, Division of Psychiatry, University College London, UK; Jennifer Mutch, MRCPsych, Community Learning Disability Service, Lynebank Hospital, Scotland, UK; Louise Marston, PhD, Primary Care and Population Health, University College London, UK; David Osborn, PhD MRCPsych, Division of Psychiatry, University College London, UK; Angela Hassiotis $\mathbb{D}^{\mathrm{D}}$, PhD FRCPsych, Division of Psychiatry, University College London, UK

Correspondence: Angela Hassiotis. Email: a.hassiotis@ucl.ac.uk

First received 29 Mar 2020, final revision 24 Sep 2020, accepted 16 Oct 2020

\section{Supplementary material}

Supplementary material is available online at https://doi.org/10.1192/bjo.2020.135 


\section{Data availability}

This database of anonymised data is accessible only to approved researchers within Camden and Islington Foundation NHS Trust and its partner research organisations.

\section{Acknowledgements}

With thanks to Nomi Werbeloff, data analyst at Camden and Islington Foundation NHS Trust, who assisted with data handling of the Clinical Record Interactive Search system.

\section{Author contributions}

A.H. formulated the research question and prepared the article with R.S. A.H. and D.O. designed the study. J.M. carried out data extraction and validation. R.S. carried out the analysis with oversight from L.M., who wrote the analytical plan. All authors have contributed to all iterations of the manuscript and its intellectual content.

\section{Funding}

This work was supported by a National Institute of Health Research grant (DRF-2016-09-140) to R.S. The funders had no role in study design, data collection and analysis, decision to publish or preparation of the manuscript.

\section{Declaration of interest}

None.

\section{References}

1 Hofvander B, Delorme R, Chaste P, Nydén A, Wentz E, Ståhlberg O, et al. Psychiatric and psychosocial problems in adults with normal-intelligence autism spectrum disorders. BMC Psychiatry 2009; 9(1): 35.

2 Cooper S-A, Smiley E, Morrison J, Williamson A, Allan L. Mental ill-health in adults with intellectual disabilities: prevalence and associated factors. $\mathrm{Br} \mathrm{J}$ Psychiatry 2007; 190(1): 27-35.

3 National Health Service (NHS) England. NHS Long Term Plan. NHS England, 2019 (https://www.england.nhs.uk/long-term-plan/).

4 National Health Service (NHS) England. Care and Treatment Reviews: Policy and Guidance. NHS England, 2015 (https://www.england.nhs.uk/publication/careand-treatment-reviews-policy-and-guidance/).
5 Werbeloff N, Osborn DP, Patel R, Taylor M, Stewart R, Broadbent M, et al. The Camden \& Islington Research Database: using electronic mental health records for research. PLOS One 2018; 13(1): e0190703.

6 Hall IS, Parkes C, Smuels S, Hassiotis A. Working across boundaries: clinical outcomes for an integrated mental health service for people with intellectual disabilities. J Intellect Disabil Res 2006; 50(8): 598-607.

7 World Health Organization. The ICD-10 Classification of Mental and Behavioural Disorders: Clinical Descriptions and Diagnostic Guidelines. World Health Organization, 1992 (https://www.who.int/classifications/icd/en/bluebook.pdf).

8 National Statistics. English Indices of Deprivation 2019. Ministry of Housing, Communities \& Local Government, 2019 (https://www.gov.uk/government/ statistics/english-indices-of-deprivation-2019).

9 Wing J, Beevor A, Curtis R, Park S, Hadden J, Burns A. Health of the Nation Outcome Scales (HoNOS): research and development. Br J Psychiatry 1998; 172(1): $11-8$

10 National Health Service (NHS) England. Building the Right Support. NHS England, 2015 (https://www.england.nhs.uk/wp-content/uploads/2015/10/ld-nat-impplan-oct15.pdf).

11 Hatton C. Transforming Care Report Card 1: People Being Admitted to Inpatient Units. 2019 (https://chrishatton.blogspot.com/2019/03/transforming-care-reportcard-1-people.html).

12 Department of Health. Valuing People: A New Strategy for Learning Disability for the 21st Century. Department of Health, 2001 (https://assets.publishing. service.gov.uk/government/uploads/system/uploads/attachment_data/file/ 250877/5086.pdf)

13 Bhavsar V, Maccabe JH, Hatch S, Hotopf M, Boydell J, McGuire P. Subclinical psychotic experiences and subsequent contact with mental health services. BJPsych Open 2017; 3: 64-70.

14 Oliver C, Piachaud J, Tyrer P, Regan A, Dack M, Alexander R, et al. Randomized controlled trial of assertive community treatment in intellectual disability: the TACTILD study. J Intellect Disabil Res 2005; 49(7): 507-15.

15 Neijmeijer LJ, Korzilius HPLM, Kroon H, Nijman HLI, Didden R. Flexible assertive community treatment for individuals with a mild intellectual disability or borderline intellectual functioning: results of a longitudinal study in the Netherlands. J Intellect Disabil Res 2019; 63(8): 1015-22.

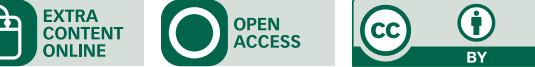

